\title{
A!
}

This is an electronic reprint of the original article.

This reprint may differ from the original in pagination and typographic detail.

Chen, Ke; Zhou, Xu; Cheng, Xu; Qiao, Ruixi; Cheng, Yi; Liu, Can; Xie, Yadian; Yu, Wentao; Yao, Fengrui; Sun, Zhipei; Wang, Feng; Liu, Kaihui; Liu, Zhongfan

Graphene photonic crystal fibre with strong and tunable light-matter interaction

Published in:

Nature Photonics

DOI:

$10.1038 / s 41566-019-0492-5$

Published: 12/08/2019

Document Version

Peer reviewed version

Please cite the original version:

Chen, K., Zhou, X., Cheng, X., Qiao, R., Cheng, Y., Liu, C., Xie, Y., Yu, W., Yao, F., Sun, Z., Wang, F., Liu, K., \& Liu, Z. (2019). Graphene photonic crystal fibre with strong and tunable light-matter interaction. Nature

Photonics, 13, 754-759. https://doi.org/10.1038/s41566-019-0492-5

This material is protected by copyright and other intellectual property rights, and duplication or sale of all or part of any of the repository collections is not permitted, except that material may be duplicated by you for your research use or educational purposes in electronic or print form. You must obtain permission for any other use. Electronic or print copies may not be offered, whether for sale or otherwise to anyone who is not an authorised user. 


\section{Graphene Photonic Crystal Fibre with Strong and Tunable Light-Matter Interaction}

3 Ke Chen ${ }^{1,2 \#}, \mathrm{Xu} \mathrm{Zhou}^{3 \#}, \mathrm{Xu}_{\mathrm{Cheng}}{ }^{3 \#}$, Ruixi Qiao ${ }^{3}$, Yi Cheng ${ }^{1,4}$, Can Liu ${ }^{3}$, Yadian Xie ${ }^{1,4}$, Wentao Yu ${ }^{3}$,

$4 \quad$ Fengrui $\mathrm{Yao}^{3}$, Zhipei $\mathrm{Sun}^{5}$, Feng Wang ${ }^{6}$, Kaihui Liu ${ }^{3 *}$, Zhongfan Liu ${ }^{1,4^{*}}$

$5{ }^{1}$ Centre for Nanochemistry, Beijing Science and Engineering Centre for Nanocarbons, Beijing National

6 Laboratory for Molecular Sciences, College of Chemistry and Molecular Engineering, Peking

$7 \quad$ University, Beijing 100871, China

$8{ }^{2}$ Institute of Micro/Nano Photonic Materials and Applications, School of Physics and Electronics, $9 \quad$ Henan University, Kaifeng 475004, China

103 State Key Laboratory for Mesoscopic Physics, Academy for Advanced Interdisciplinary Studies, 11 School of Physics, Peking University, Beijing 100871, China

$12{ }^{4}$ Beijing Graphene Institute (BGI), Beijing 100095, China

$24 \quad$ " These authors contributed equally to this work

25 *Correspondence: zfliu@pku.edu.cn; khliu@pku.edu.cn FI-00076, Aalto, Finland 17 8 9

.

${ }^{5}$ Department of Electronics and Nanoengineering and QTF Centre of Excellence, Aalto University,

${ }^{6}$ Department of Physics, University of California at Berkeley, Berkeley, CA 94720, USA 
The integration of photonic crystal fibre (PCF) with various functional materials has greatly expanded the application regimes of optical fibre ${ }^{1-12}$. The emergence of graphene excites new opportunities by combining with PCF, allowing for electrical tunability, broadband optical response and all-fibre integration ability ${ }^{13-18}$. However, the previous demonstrations are typically limited to the sample level of micron size, far behind the requirement of real applications for the metre-scale material level. Here, we demonstrate a new hybrid material of graphene photonic crystal fibre (Gr-PCF) with length up to half a metre by chemical vapour deposition method. The Gr-PCF shows strong light-matter interaction with $\sim 8 \mathrm{~dB} \cdot \mathrm{cm}^{-1}$ attenuation. In addition, the Gr-PCF-based electro-optic modulator demonstrates broadband response (1150 - $1600 \mathrm{~nm})$ and large modulation depth $\left(\sim 20 \mathrm{~dB} \cdot \mathrm{cm}^{-1}\right.$ at $\left.1550 \mathrm{~nm}\right)$ under low gate voltage of $\sim 2$ volts. Our results could enable industrial-level graphene applications based on the Gr-PCF, and suggest an infusive platform of two-dimensional material-PCF.

Graphene is a promising material in photonic and optoelectronic applications due to its superior properties of high carrier mobility, broadband optical response and facile electrical tunability originating from its unique linear dispersion of massless Dirac fermions ${ }^{19-28}$. Although the light-matter interaction in graphene normalized by its atomic thickness $(0.34 \mathrm{~nm})$ is quite strong, the measurable interaction is in fact quite weak (only $\sim 2.3 \%$ light absorption) ${ }^{29}$. To greatly enhance light-graphene interaction, many efforts have been devoted to combine graphene flakes with well-designed optical structures, such as gratings, waveguides and microcavities ${ }^{30-34}$, however, all those hybrid structures have still stayed at sample level of micron size, rather than material level of metre size, which limits their massive applications. Therefore, there exists great demand to develop new methods for massive production on graphene-based optical structures for material-level applications.

Optical fibre provides the highest-quality optical waveguide for information communication and photon manipulation, and it has been massively manufactured at kilometre length scale. PCF represents the most important advance of optical fibre in the last twenty years and possesses extremely rich functions beyond traditional optical fibre in the exciting applications of endlessly single-mode fibres, supercontinuum lasers, frequency combs, optical soliton propagation, high-power pulse delivery and so $\mathrm{on}^{1-7}$. Especially, PCF with ingenious porous structure opens up the hard-won opportunity of filling various materials, ranging from gases, liquids, solids to liquid crystals, to expand its great new 
functionalities in mode-locked fibre lasers, laser frequency conversion, surface plasmon generation, stimulated Raman scattering and in-fibre thermal- or electro-optic devices ${ }^{8-15}$. The rise of two-dimensional (2D) graphene naturally excites the keen interests in combining PCF with graphene. Such graphene-PCF complex can occupy several unique advantages: $(i)$ the flexibility of graphene facilitates its tight attachment to the hole walls of PCF; (ii) the atomic thickness of graphene keeps the PCF structure and main optical function intact, and (iii) the distinct properties of graphene bring the unique functions that can't be realized by any other conventional materials. Indeed, great efforts have been delivered to fabricate graphene-optical fibre complex by transferring graphene flakes on side-polished or tapered normal optical fibres ${ }^{16-18}$, or filling them into the holes of $\mathrm{PCFs}^{13-15}$. However, all these attempts are only at sample level of micron length with small interaction area and harmful treating on fibre modes, and it is still lacking an efficient and nonharmful manufacture strategy for the massive production at metre-sized material level for graphene-PCF complex with large interaction area and intact structure yet.

In this work, we report the production of the new hybrid material of Gr-PCF with half-metre length by direct chemical vapour deposition (CVD) growth method, which was previously believed extremely challenging due to the lack of metal catalyst and the difficulties in gas flow control along the long micron-sized holes in silica PCF. Our success herein benefits from our extensive experience in the growth of graphene glass ${ }^{35}$ and the well control of molecular gas flow in the confined space ${ }^{36}$. With this new Gr-PCF material, we realized greatly enhanced light-matter interaction between graphene and core-guided light with $\sim 8 \mathrm{~dB} \cdot \mathrm{cm}^{-1}$ transmission attenuation. We also demonstrated the tunable light-matter interaction by electrically gating graphene through ionic liquid, with large modulation depth $\left(\sim 20 \mathrm{~dB} \cdot \mathrm{cm}^{-1}\right.$ at $\left.1550 \mathrm{~nm}\right)$ and broadband wavelength response $(1150-1600 \mathrm{~nm})$ under low gate voltage ( $\sim 2$ volts). And the fully enhanced and tunable light-matter interaction in Gr-PCF material indicates its great potentials in the all-fibre integration devices. Our results pave a new way for hybrid optical fibre manufacturing and suggest an exciting material platform of 2D materials-integrated fibre with unprecedented function tunability in both linear and nonlinear optics.

In our experiment, Gr-PCF was grown with methane $\left(\mathrm{CH}_{4}\right)$ as carbon feedstock flowing through the PCF's narrow holes ( $4 \mu \mathrm{m}$ in diameter) under $\sim 1100{ }^{\circ} \mathrm{C}$ with controlled pressure (Fig. 1a). After the growth, the PCF can keep its structure intact which consists of a solid silica core and a surrounding 
cladding region with a patterned array of holes (Fig. 1b), while graphene is grown on both the outer surface and inner hole walls of PCF. The fully-covered graphene on the outer surface was unambiguously evidenced by the Raman mapping (Fig. 1c and Supplementary Fig. 1). Representative Raman spectrum (Fig. 1d) shows sharp G- and 2D-mode peaks, revealing the high quality of graphene (The observable D peak mainly comes from the defective grain boundaries between graphene domains $)^{37}$. The fully-covered graphene on the inner hole walls can be firstly indicated by the darker optical contrast of Gr-PCF than bare PCF, due to the light absorption by graphene (Fig. 1e,f). Direct evidence of graphene growth inside PCF can be given by the observation of tube-like graphene frameworks that tightly attach on the hole walls and protrude out the holes when one breaks the Gr-PCF (Fig. 1g and Supplementary Fig. 2). Furthermore, the inner graphene films can be directly obtained by dissolving the fibre silica in hydrofluoric acid (the graphene film on the outer surface was first removed by air-plasma treatment). The cylindroid graphene films from Gr-PCF collapse into ribbon films on silicon substrate, with an average layer thickness of $\sim 2.0 \mathrm{~nm}$ as measured by atomic force microscope (AFM) (Fig. 1h,i). Here we note that the interlayer distance is relatively larger (estimated as $1.0-1.5 \mathrm{~nm}$ ) than its intrinsic interlayer distance $(0.34 \mathrm{~nm})$ in these collapsed ribbons, likely due to the wrinkles formed during the etching treatment. The selected-area electron diffraction (SAED) in Fig. $1 \mathrm{j}$ demonstrates the polycrystalline structure of the graphene film, as the selected-area aperture size (200 $\mathrm{nm})$ is several times of graphene domain size $(\sim 50 \mathrm{~nm})$. However, the high-resolution transmission electron microscopy (HRTEM) image on individual graphene domain shows perfect graphene lattice structure (Moiré pattern of bilayer as an example in Fig. 1k). More transmission electron microscopy (TEM) characterizations reveal that the graphene thickness can be controlled between one to ten layers by the growth time (Supplementary Fig. 3).

One great concern about the Gr-PCF growth is whether the graphene can be homogenously distributed on the micron-sized hole walls along the long fibre, as gas flow in narrow space will have huge viscous force. To clarify this point, we carried out control experiments with atmosphere pressure chemical vapour deposition (APCVD, Fig. 2a) and low-pressure chemical vapour deposition (LPCVD, pressure of $0.5-1.0 \mathrm{kPa}$, Fig. 2b). According to viscous flow model ${ }^{38}$ (Supplementary Note 1), the mean free path of carbon precursors (estimated as $\sim 0.4 \mu \mathrm{m}$ ) is much shorter than the fibre hole diameter $(\sim 4$ $\mu \mathrm{m})$ under the atmosphere pressure in APCVD process. In this situation, the carbon precursor feeding is 
limited by the mass transfer ${ }^{36}$. According to experimental Raman data of graphene ribbons etched from the holes of Gr-PCF with $\sim 8 \mathrm{~cm}$ length, the obvious variation of 2D- to G-mode Raman (intensity ratio $\left.\mathrm{I}_{2 \mathrm{D}} / \mathrm{I}_{\mathrm{G}}, 0.6-2.1\right)$ and full width at half maximum of $2 \mathrm{D}$-mode $\left(\mathrm{FWHM}_{2 \mathrm{D}}, 31-71 \mathrm{~cm}^{-1}\right)$ demonstrate the nonuniform thickness along the fibre axis (Fig. 2c,d, APCVD panels). This result is consistent with optical contrast observation that the fibre became darker along the gas flow direction (Supplementary Fig. 4, lower fibre). It means the graphene film thickness increases along the gas flow, originating from the increased active carbon species during the long-time thermal decomposition in the downstream positions. Whereas, in LPCVD process, the gas flow approaches a free molecular flow situation and the mass-diffusion process becomes negligible ${ }^{36,38}$. In this case, the mean free path (estimated as $>40 \mu \mathrm{m}$ ) is much larger than hole size of PCF. Then elapse time ( $\sim 15 \mathrm{~s})$ of gaseous molecules going through the long holes of PCF in LPCVD process is much shorter than that in APCVD process ( $\sim 175 \mathrm{~s})$. It is facile to realize that the graphene films on the inner hole walls grow with uniform thickness along the fibre axis, as revealed by the Raman spectra with negligible fluctuation of $\mathrm{I}_{2 \mathrm{D}} / \mathrm{I}_{\mathrm{G}}$ ratio $(\sim 1.4)$ and $\mathrm{FWHM}_{2 \mathrm{D}}$ $\left(\sim 48 \mathrm{~cm}^{-1}\right)$ (Fig. $2 \mathrm{c}, \mathrm{d}$, LPCVD panels), as well as the uniform optical imaging contrast along the whole fibre (Supplementary Fig. 4, upper fibre). Here we note that the intensity ratio of D- to G-mode Raman $\left(\mathrm{I}_{\mathrm{D}} / \mathrm{I}_{\mathrm{G}}\right)$ has much larger value in LPCVD, revealing smaller graphene domain size ${ }^{37}$, which originates from the lower carbon feedstocks, corresponding to the slower growth rate and the more nucleation centres under molecular flow. Using current furnace with heating zone of $\sim 60 \mathrm{~cm}$, we can readily grow Gr-PCF with uniform thickness and length up to $50 \mathrm{~cm}$ (Fig. 1e).

In principle, one would expect that the light-graphene interaction in Gr-PCF would be greatly enhanced as the atomic thickness of graphene wouldn't destroy the fundamental propagating mode. This assumption was checked by simulating the light electric field distribution of fundamental guiding mode with the full-vector finite element method ${ }^{30}$. In the simulation, the graphene film directly interacts with light field by evanescent wave coupling at the hole walls adjacent to the fibre core (Fig. 3a). Similar to the distribution in bare PCF (Supplementary Fig. 5d), the light in Gr-PCF is mainly confined in the fibre core and about one tenth of the electric field to that at fibre core centre is interacting with graphene on the innermost hole walls (Fig. 3b). The light-graphene interaction can be first indicated by the $3 \%$ kink in the normalized radial electric filed distribution, which originates from refractive index difference between graphene/silica and air. The significantly enhanced light-graphene interaction in Gr-PCF can be 
quantitatively measured by the propagated light intensity evolution along the fibre. In a bare PCF of $\sim 4$ cm long, there is no observable attenuation $\left(<0.01 \mathrm{~dB} \cdot \mathrm{cm}^{-1}\right)$ at all (Fig. 3c, purple dots). However, in Gr-PCF, a strong attenuation of $8.3 \mathrm{~dB} \cdot \mathrm{cm}^{-1}$ is observed (Fig. 3c, cyan dots, fitted slope), in striking contrast with only $0.1 \mathrm{~dB}$ of suspended monolayer graphene. The attenuation coefficient value of this Gr-PCF is equivalent to average graphene layer number of $\sim 1.5$ (Fig. 3c, line and Supplementary Fig. 5i). Here the 0.5 layer comes from the partial second graphene layers grown on the first graphene layer film. The greatly enhanced light-graphene interaction in Gr-PCF can be qualitatively understood by the tremendous enlargement of effective interaction area and length during the multiple reflection of light propagation along the fibre axis (Fig. 3c, lower panel). It is worth noting that the light-graphene interaction strength can be tuned by the PCF geometries, such as the air-hole diameter and the hole pitch size (Supplementary Fig. 6).

One of the unique merits of 2D graphene is the unprecedented electrical tunability in light-matter interaction by shifting its Fermi level $\left(E_{\mathrm{F}}\right)^{21,26}$. This tunability can be used to exploit the Gr-PCF as an in-line electro-optic modulator with intensity modulation in all-fibre communication networks (testing setup in Supplementary Fig. 7). Note that our Gr-PCF shows a stable and negligible loss $(<0.1 \mathrm{~dB})$ in coupling with single mode fibres. Here, we demonstrate an electro-optic modulator consisting of a short segment $(0.5-1.5 \mathrm{~cm})$ of Gr-PCF with ionic liquid (DEME-TFSI) filled inside the holes (Fig. 4a). The graphene on the hole walls of Gr-PCF connects electrically with an electrode on the outside graphene surface (the as-grown graphene films in the whole fibre are all connected). Ionic liquid is then fully filled in the fibre holes and contacts with another electrode. When gate voltage between graphene and ionic liquid is applied, electrical double layer (EDL) will form at graphene-ionic liquid interface and dope graphene efficiently under low gate voltage of several volts (Fig. 4b and Supplementary Fig. $8)^{26,39,40}$. Further simulation reveals an additional advantage of ionic liquid filling that it can increase the light-graphene interaction from 5 to $24 \mathrm{~dB} \cdot \mathrm{cm}^{-1}$ for monolayer Gr-PCF by increasing the mode field area of core-guided light (Supplementary Fig. 5), as ionic liquid has a refractive index (1.42) close to that of the PCF material (silica, 1.44).

By electrically tuning graphene Fermi level via ionic liquid gating, the interband transition absorption can be tuned at "On" or "Off" state when $E_{\mathrm{F}}$ is smaller or larger than half photon energy of $\hbar \omega / 2$, where $\omega$ and $\hbar \omega$ are the angular frequency and energy of a photon, respectively(Fig. $4 \mathrm{c})^{21,30}$. This 
absorption-based electro-optic modulator operates at very low voltage (within $2 \mathrm{~V}$ ) due to the efficient electric double layer gating, and a broadband spectral response from 1150 to $1600 \mathrm{~nm}$ due to the linear Dirac band structure of graphene (Fig. 4d). In particular, at the fibre-optic communication O- (1310 nm) and $\mathrm{C}$-wavelength bands $(1550 \mathrm{~nm})$, the modulation depths can respectively reach $\sim 13$ and $\sim 20 \mathrm{~dB} \cdot \mathrm{cm}^{-1}$ at gate voltage of $-1.8 \mathrm{~V}$ (Fig. 4e). The relatively lower gating efficiency at positive gate voltage region is attributed to the lower capacitance of our ionic liquid at electron doping side, which is consistent with the electrical device measurement (Supplementary Fig. 8). The performance of our current modulator can be further improved. Firstly, it has insertion loss of $\sim 6 \mathrm{~dB} / \mathrm{cm}$ in the "On" state, which can be much lower in principle if one grows very high-quality monolayer graphene on the inside walls of PCF. Secondly, it has slow switching speed of $\sim 16$ Hz (Supplementary Fig. 9) which can be improved (e.g., few $10 \mathrm{~s} \mathrm{GHz}^{17,31}$ ) if one can achieve optimized structures, such as $\mathrm{Gr} / \mathrm{hBN} / \mathrm{Gr}-\mathrm{PCF}^{41}$. Nevertheless, the Gr-PCF modulator has high potential to be integrated with other optical devices in all-fibre system where light can be synchronically transmitted, modulated and detected inside fibre link without the aid of discrete devices in the future ${ }^{42}$.

In summary, we demonstrated an ingenious CVD route to achieve a new material of Gr-PCF. The uniform and high-quality graphene on both the outer surface and inner hole walls of PCF with length up to half a metre was realized by low pressure growth with controlled molecular flow. Such Gr-PCF material exhibits a strong and tunable light-matter interaction and presents excellent performance as a broadband electro-optic modulation under low gate voltage. As added convenient electrical tunability, kept PCF waveguide mode intact and enhanced light-graphene interactions in both linear and nonlinear optical regime, Gr-PCF enables the potential advent of novel fibre-optic devices such as electrically-tunable mode-locked all-fibre lasers, gate-controllable wavelength-independent nonlinear wavelength converters, tunable broadband polarizers and optical limiters in the near future. In addition, our growth strategy can open up a new direction for massive production of other $2 \mathrm{D}$ crystals based all-fibre devices, targeting to the next-generation optical fibres with various new functionalities.

\section{Online content}

Any methods, additional references, Nature Research reporting summaries, source data, statements of data availability and associated accession codes are available at https://doi.org/xxx/ xxx Received: xx xx xxx; Accepted: xx xx xxx; 
201

202

203

204

205

206

207

208

209

210

211

212

213

214

215

216

217

218

219

220

221

222

223

224

225

226

227

228

229

230

231

232

233

234

235

236

237

238

239

\section{References}

1 Russell, P. Photonic crystal fibers. Science 299, 358-362 (2003).

2 Knight, J. C. Photonic crystal fibres. Nature 424, 847-851 (2003).

3 Ouzounov, D. G. et al. Generation of megawatt optical solitons in hollow-core photonic band-gap fibers. Science 301, 1702-1704 (2003).

4 Bartels, R. A. et al. Generation of spatially coherent light at extreme ultraviolet wavelengths. Science 297, 376-378 (2002).

5 Couny, F., Benabid, F., Roberts, P. J., Light, P. S. \& Raymer, M. G. Generation and photonic guidance of multi-octave optical-frequency combs. Science 318, 1118-1121 (2007).

6 Dudley, J. M. \& Taylor, J. R. Ten years of nonlinear optics in photonic crystal fibre. Nat. Photon. 3, 85-90 (2009).

7 Jiang, X. et al. Deep-ultraviolet to mid-infrared supercontinuum generated in solid-core ZBLAN photonic crystal fibre. Nat. Photon. 9, 133-139 (2015).

8 Benabid, F., Knight, J. C., Antonopoulos, G. \& Russell, P. S. J. Stimulated Raman scattering in hydrogen-filled hollow-core photonic crystal fiber. Science 298, 399-402 (2002).

9 Abouraddy, A. F. et al. Towards multimaterial multifunctional fibres that see, hear, sense and communicate. Nat. Mater. 6, 336-347 (2007).

$10 \mathrm{He}, \mathrm{R}$. R. et al. Integration of gigahertz-bandwidth semiconductor devices inside microstructured optical fibres. Nat. Photon. 6, 174-179 (2012).

11 Kottig, F. et al. Mid-infrared dispersive wave generation in gas-filled photonic crystal fibre by transient ionization-driven changes in dispersion. Nat. Commun. 8, 813 (2017).

12 Rein, M. et al. Diode fibres for fabric-based optical communications. Nature 560, 214-218 (2018).

13 Choi, S. Y. et al. Graphene-filled hollow optical fiber saturable absorber for efficient soliton fiber laser mode-locking. Opt. Express 20, 5652-5657 (2012).

14 Martinez, A. \& Sun, Z. P. Nanotube and graphene saturable absorbers for fibre lasers. Nat. Photon. 7, 842-845 (2013).

15 Lin, Y.-H., Yang, C.-Y., Liou, J.-H., Yu, C.-P. \& Lin, G.-R. Using graphene nano-particle embedded in photonic crystal fiber for evanescent wave mode-locking of fiber laser. Opt. Express 21, 16763-16776 (2013).

16 Bao, Q. L. et al. Broadband graphene polarizer. Nat. Photon. 5, 411-415 (2011).

17 Li, W. et al. Ultrafast All-Optical Graphene Modulator. Nano Lett. 14, 955-959 (2014).

18 Lee, E. J. et al. Active control of all-fibre graphene devices with electrical gating. Nat. Commun. 6, 6851 (2015).

19 Zhang, Y. B., Tan, Y. W., Stormer, H. L. \& Kim, P. Experimental observation of the quantum Hall effect and Berry's phase in graphene. Nature 438, 201-204 (2005).

20 Novoselov, K. S. et al. Two-dimensional gas of massless Dirac fermions in graphene. Nature 438, 197-200 (2005).

21 Wang, F. et al. Gate-variable optical transitions in graphene. Science 320, 206-209 (2008).

22 Xia, F. N., Mueller, T., Lin, Y. M., Valdes-Garcia, A. \& Avouris, P. Ultrafast graphene photodetector. 
Nat. Nanotechnol. 4, 839-843 (2009).

23 Bonaccorso, F., Sun, Z., Hasan, T. \& Ferrari, A. C. Graphene photonics and optoelectronics. Nat. Photon. 4, 611-622 (2010).

24 Grigorenko, A. N., Polini, M. \& Novoselov, K. S. Graphene plasmonics. Nat. Photon. 6, 749-758 (2012).

25 Gan, X. T. et al. Chip-integrated ultrafast graphene photodetector with high responsivity. Nat. Photon. 7, 883-887 (2013).

26 Polat, E. O. \& Kocabas, C. Broadband Optical Modulators Based on Graphene Supercapacitors. Nano Lett. 13, 5851-5857 (2013).

27 Guo, Q. et al. Efficient electrical detection of mid-infrared graphene plasmons at room temperature. Nat. Mater. 17, 986-992 (2018).

28 Romagnoli, M. et al. Graphene-based integrated photonics for next-generation datacom and telecom. Nat. Rev. Mater. 3, 392-414 (2018).

29 Nair, R. R. et al. Fine structure constant defines visual transparency of graphene. Science 320, 1308-1308 (2008).

30 Liu, M. et al. A graphene-based broadband optical modulator. Nature 474, 64-67 (2011).

31 Phare, C. T., Lee, Y. H. D., Cardenas, J. \& Lipson, M. Graphene electro-optic modulator with 30 GHz bandwidth. Nat. Photon. 9, 511-514 (2015).

32 Lin, H. T. et al. Chalcogenide glass-on-graphene photonics. Nat. Photon. 11, 798-805 (2017).

33 Sorianello, V. et al. Graphene-silicon phase modulators with gigahertz bandwidth. Nat. Photon. 12,40-44 (2018).

34 Yao, B. C. et al. Gate-tunable frequency combs in graphene-nitride microresonators. Nature 558, 410-414 (2018).

35 Sun, J. Y. et al. Graphene Glass from Direct CVD Routes: Production and Applications. Adv. Mater. 28, 10333-10339 (2016).

36 Wang, H. et al. Surface Monocrystallization of Copper Foil for Fast Growth of Large Single-Crystal Graphene under Free Molecular Flow. Adv. Mater. 28, 8968-8974 (2016).

37 Sato, K. et al. D-band Raman intensity of graphitic materials as a function of laser energy and crystallite size. Chem. Phys. Lett. 427, 117-121 (2006).

38 Knudsen, M. The kinetic theory of gases (Methuen, London, 1952).

39 Ye, J. T. et al. Liquid-gated interface superconductivity on an atomically flat film. Nat. Mater. 9, 125-128 (2010).

40 Fujimoto, T. \& Awaga, K. Electric-double-layer field-effect transistors with ionic liquids. Phys. Chem. Chem. Phys. 15, 8983-9006 (2013).

41 Yang, W. et al. Epitaxial growth of single-domain graphene on hexagonal boron nitride. Nat. Mater. 12, $792-797$ (2013).

42 Yamashita, S. Nonlinear optics in carbon nanotube, graphene, and related 2D materials. Apl. Phys. Lett. Photon. 4, 034301 (2019). 


\section{Acknowledgements}

This work was supported by National Key R\&D Program of China (2016YFA0200103, 2016YFA0300903, 2016YFA0300804), Beijing Graphene Innovation Program (Z181100004818003, Z161100002116028), NSFC (51432002，51520105003，51502077，51522201，11474006), Beijing Municipal Science \& Technology Commission (Z181100004218006), National Equipment Program of China (ZDYZ2015-1), Postdoctoral Innovative Personnel Support Program (BX20180013), The Science and Technology Development Project of Henan Province (182102210029), Zhongyuan Thousand Talents Program of Henan Province, Young Talents Program of Henan University for support. Director, Office of Science, Office of Basic Energy Sciences, Materials Sciences and Engineering Division of the U.S. Department of Energy under Contract No. DE-AC02-05-CH11231 (SP2 program), Academy of Finland (276376, 295777, 312297, and 314810), Academy of Finland Flagship Programme (320167, PREIN), the ERC (834742), and the European Union's Horizon 2020 research and innovation program (820423, S2QUIP)

\section{Author contributions}

Z.L. and K.L. supervised the project. Z.L. and K.C. conceived the material growth. K.L. and X.Z. conceived the optical measurement. K.C. and X.Z. carried out the material growth experiment and optical device measurements. X.C. performed theoretical modelling. K.C., R.Q., Y.C., Y.X., X.Z., C.L. and F.Y. conducted SEM, TEM, AFM and Raman characterizations. W.Y. suggested the optical experiments. F.Y. programmed the measurement software. Z.S. and F.W. suggested the modulator. All authors contributed to the scientific discussion and writing of the manuscript.

\section{Competing interests}

The authors declare no competing financial interests.

\section{Additional information}

Supplementary information is available is available for this paper at https://dio.org/xxx/xxx.

Reprints and permissions information is available at www.nature.com/reprints.

Correspondence and requests for materials should be addressed to Z.L. (for materials) and K.L. (for optics).

Publisher's note: Springer Nature remains neutral with regard to jurisdictional claims in published maps and institutional affiliations.

(C) The Author(s), under exclusive licence to Springer Nature Limited 2019 
309 Fig. 1 | Growth and characterization of Gr-PCF. a, Schematics of Gr-PCF grown by CVD method, 310 with graphene film on both outer surface and inner walls of PCF. b, SEM image of Gr-PCF end surface. 311 c,d, 2D-mode Raman intensity mapping (c) and Raman spectrum (d) of graphene at fibre end surface. e,f, 312 Optical reflection photograph (e) and transmission micrograph (f) of bare PCF and Gr-PCF. The optical 313 contrast is darker for Gr-PCF due to the light absorption by graphene. g, SEM image of a tube-like 314 graphene protruding out one hole of the fractured Gr-PCF. h, SEM image of a graphene ribbon (the 315 collapsed tube-like graphene grown on the hole wall) after dissolving the fibre silica. i, AFM image of a 316 graphene ribbon with wrinkles. The height of $\sim 2.0 \mathrm{~nm}$ is obtained along the white dash line. $\mathbf{j}, \mathbf{k}$, SAED 317 (j) and HRTEM (k) images of the graphene ribbon.

Fig. 2 | Controlled growth of uniform graphene film on the hole walls of PCF. a,b, Schematics of graphene growth on the walls of the micron-size holes of PCFs at atmosphere pressure (AP) and low pressure (LP) CVD. c, Representative Raman spectra of graphene at different positions of graphene ribbons along the gas flow under APCVD (upper panel) and LPCVD (lower panel) growth conditions. The graphene ribbons are from the hole walls after etching away the fibre silica. d, The statistics of $\mathrm{I}_{\mathrm{D}} / \mathrm{I}_{\mathrm{G}}$, $\mathrm{I}_{2 \mathrm{D}} / \mathrm{I}_{\mathrm{G}}$ and the $\mathrm{FWHM}_{2 \mathrm{D}}$ of graphene Raman peak at different positions of graphene ribbons by APCVD and LPCVD, where D, G and 2D stands for D-, G- and 2D-mode Raman peaks of graphene, respectively. 
Fig. 3 | Strong light-matter interaction in Gr-PCF. a, The simulated light electric field distribution of the fundamental guiding mode at $1550 \mathrm{~nm}$. The graphene is set on the hole wall shown in the profiles of Gr-PCF (low panel, yellow rectangle). b, The distribution of normalized electric field in Gr-PCF along horizontal radial direction (yellow horizontal line in (a)). The right panel shows the zoom-in of the dash square in left panel. The black arrow highlights the graphene position. $\mathbf{c}$, Measured optical attenuation of light propagation in bare PCF (purple dots) and Gr-PCF (cyan dots) with different fibre lengths. The attenuation coefficient is fitted as $8.3 \mathrm{~dB} \cdot \mathrm{cm}^{-1}$ of the slope. The lower panel gives the schematic of light attenuation with multiple reflection during its propagating along Gr-PCF core. The error bar is the standard deviation from the measurement of 5 samples for each length grown at the same growth conditions.

Fig. 4 | Tunable light-matter interaction in Gr-PCF. a, Schematic of a Gr-PCF-based electro-optic modulator. The gate voltage between ionic liquid and graphene controls the light transmission through Gr-PCF. b,c, The working principle of Gr-PCF electro-optic modulator where electric double layer forms at interface between graphene (dark grey rectangle) and ionic liquid (blue area). The ionic liquid-gating tunes the graphene's Fermi level and switches on and off the optical absorption in graphene. When $E_{\mathrm{F}}<\hbar \omega / 2\left(E_{\mathrm{F}}>\hbar \omega / 2\right)$, graphene absorbs (doesn't absorb) light and the modulator is working at "Off" ("On") state for light transmission in the fibre. d, Two-dimensional mapping of transmission modulation (normalized by fibre length) of Gr-PCF modulator as a function of gate voltage and optical wavelength. e, The modulation curves at 1310 and $1550 \mathrm{~nm}$ show an unambiguous transition between "On" and "Off" state with large modulation depth. 


\section{Methods}

Growth of Gr-PCFs. The PCFs $(125 \mu \mathrm{m}$ of cladding diameter and $8 \mu \mathrm{m}$ of core diameter $)$ were placed in the centre of a tube furnace (Thermo Linderberg). For APCVD process, $\mathrm{CH}_{4}(10$ sccm $)$ was introduced as a carbon feedstock with $\mathrm{Ar}(100 \mathrm{sccm})$ and $\mathrm{H}_{2}(50 \mathrm{sccm})$ at $1100{ }^{\circ} \mathrm{C}$ for hours under atmosphere pressure. For LPCVD process, $\mathrm{CH}_{4}(50 \mathrm{sccm})$ was mixed with the $\mathrm{H}_{2}(50 \mathrm{sccm})$ at $1100{ }^{\circ} \mathrm{C}$ for hours under pressure of 500 - $1000 \mathrm{~Pa}$. After the growth, the as-grown sample was naturally cooled down in the protective gases of $\mathrm{Ar}$ and $\mathrm{H}_{2}$.

Characterization of Gr-PCFs. An optical microscope (Olympus BX51) was used to obtain the optical images of Gr-PCFs. SEM images were collected by FEI Nova ${ }^{\mathrm{TM}}$ Nano-SEM430 at $5 \mathrm{kV}$. Raman spectra and mappings were taken by WITec alpha 300R-confocal Raman imaging system equipped with a 532 $\mathrm{nm}$ laser. AFM pictures and data were performed in a Bruker Dimension Icon atomic force microscope. HRTEM and SAED experiments were performed in an aberration-corrected FEI Titan Themis G2-300 at $80 \mathrm{kV}$.

Numerical modelling. Numerical simulations were processed by the RF module of COMSOL's Multiphysics software and MATLAB software. The effective refractive index of fundamental guiding mode in Gr-PCFs was calculated by finite element method in COMSOL.

Device fabrication and modulation measurement. Ti/Au $(5 / 100 \mathrm{~nm})$ electrodes were deposited on graphene film by e-beam evaporation. The ionic liquid (DEME-TFSI, Sigma-Aldrich, CAS: 464927-84-2) was injected into holes of Gr-PCF with another Ti/Au electrode connection. The two ends of Gr-PCF were connected with two single-mode optical fibres (SMFs, Corning SMF-28e+) and the gap between fibres were filled with ionic liquid. The two SMFs were aligned in line with Gr-PCF by our home-built setup (Supplementary Fig. 6), where two optical microscopic observation in different directions and multi-axis piezo-stages ensured the accurate alignment. Keithley 2400 Source Meter provided the gate voltage. Supercontinuum laser (NKT Photonics Inc.) was coupled into SMF to provide broadband light. Modulated signal was sent into a spectrograph equipped with an infrared CCD (Princeton Instruments: Acton SpectraPro® SP-2300 and Pylon-IR 1024-1.7). 


\section{Data availability}

372 The data that support the plots within this paper and other findings of this study are available from the 373 corresponding author upon reasonable request. 
a

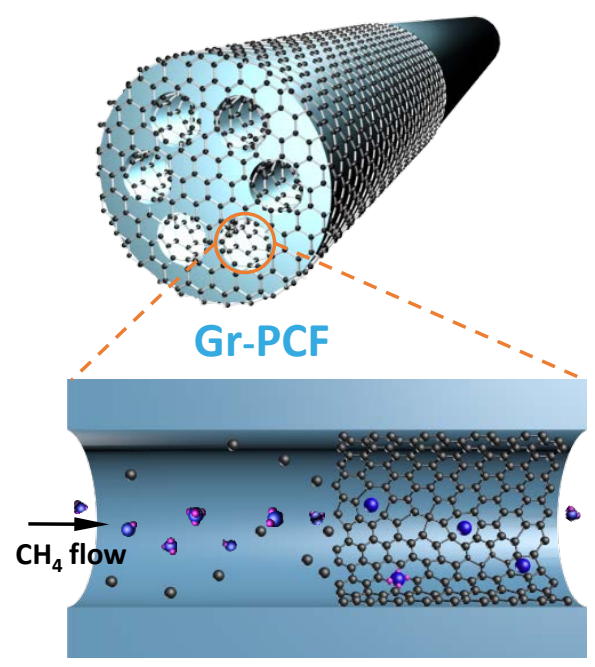

b

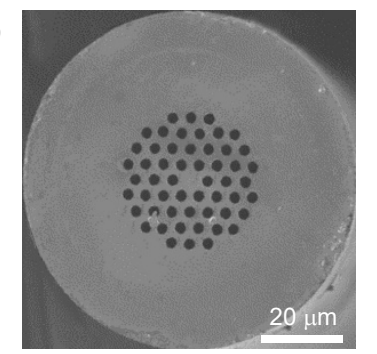

d

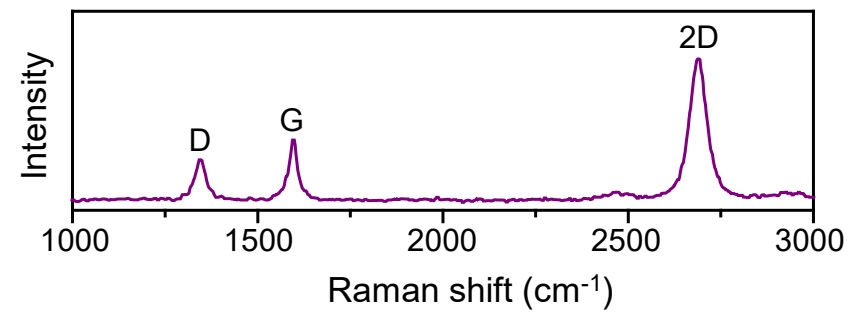

C

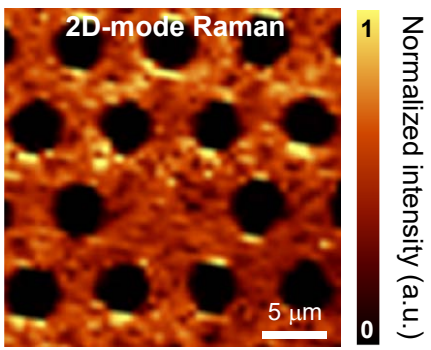

e

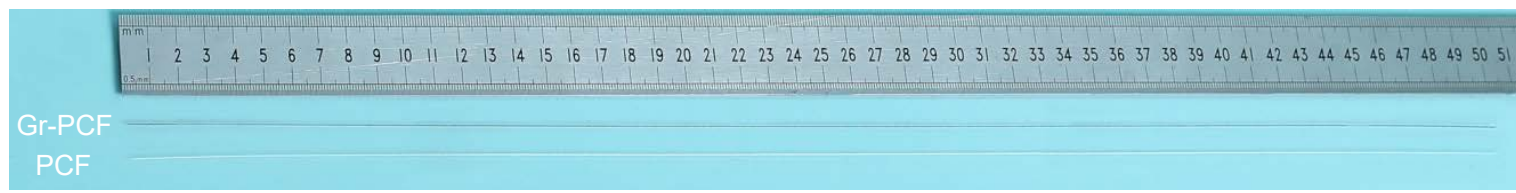

f

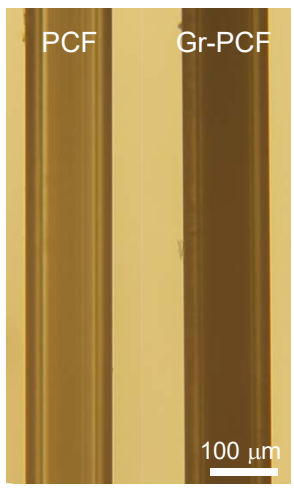

g

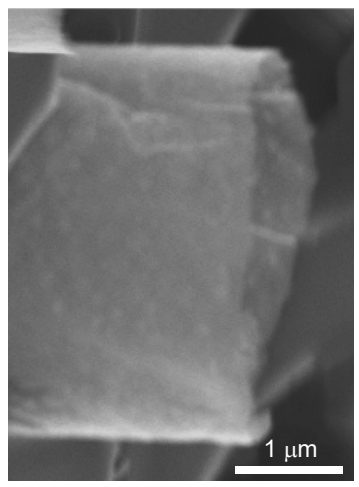

h

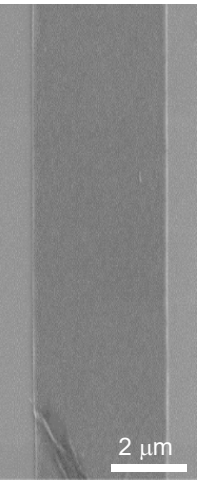

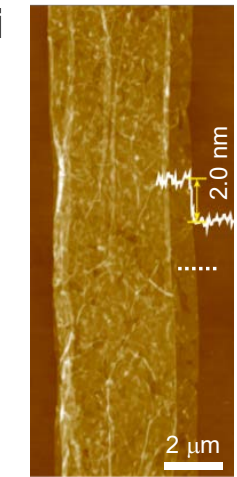

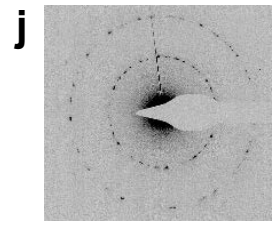

k

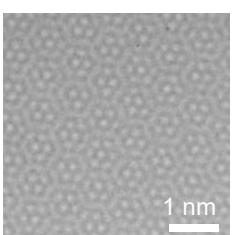

Fig.1 
a

APCVD

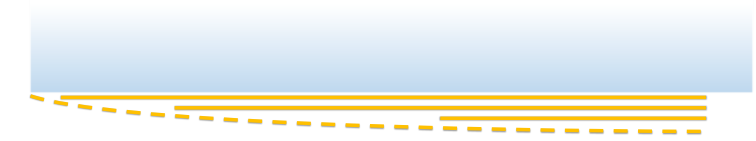

Gas flow

Viscous fluid

C
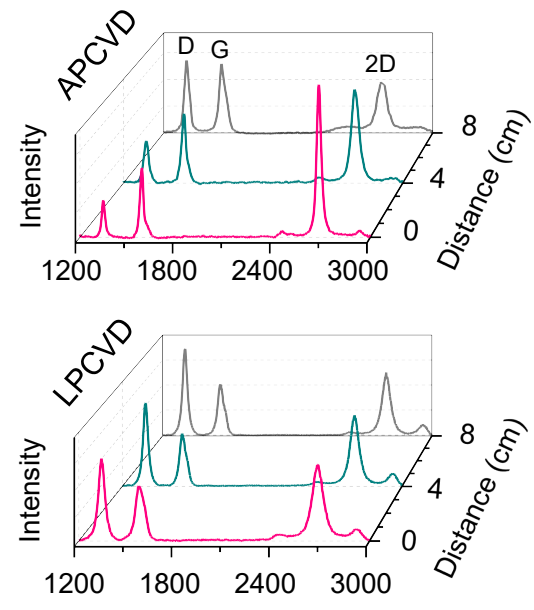

Raman shift $\left(\mathrm{cm}^{-1}\right)$ b

LPCVD

Gas flow

Molecular fluid

Gr layers

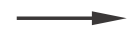

Molecular fluid

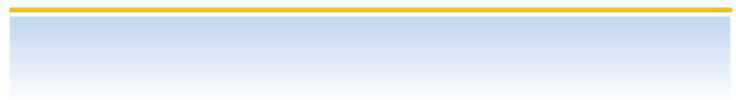

APCVD
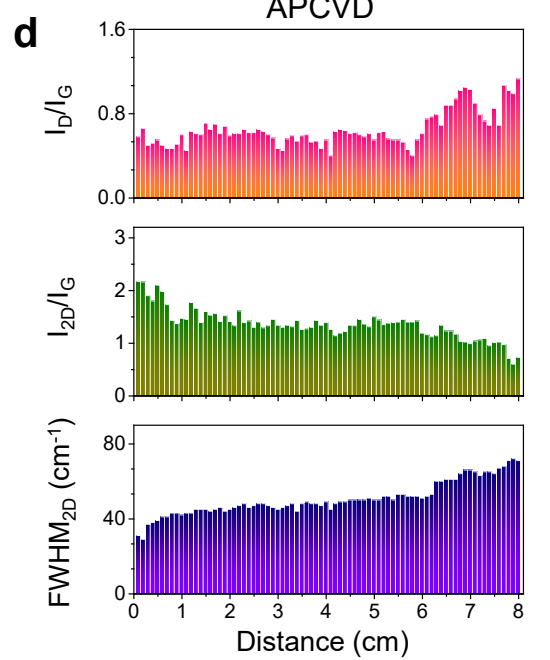

LPCVD
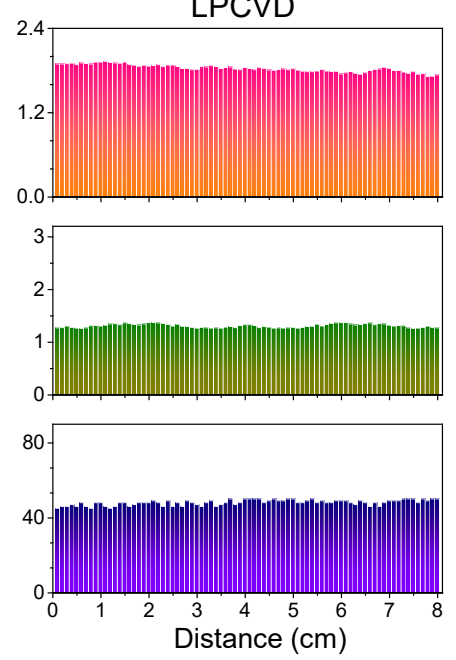

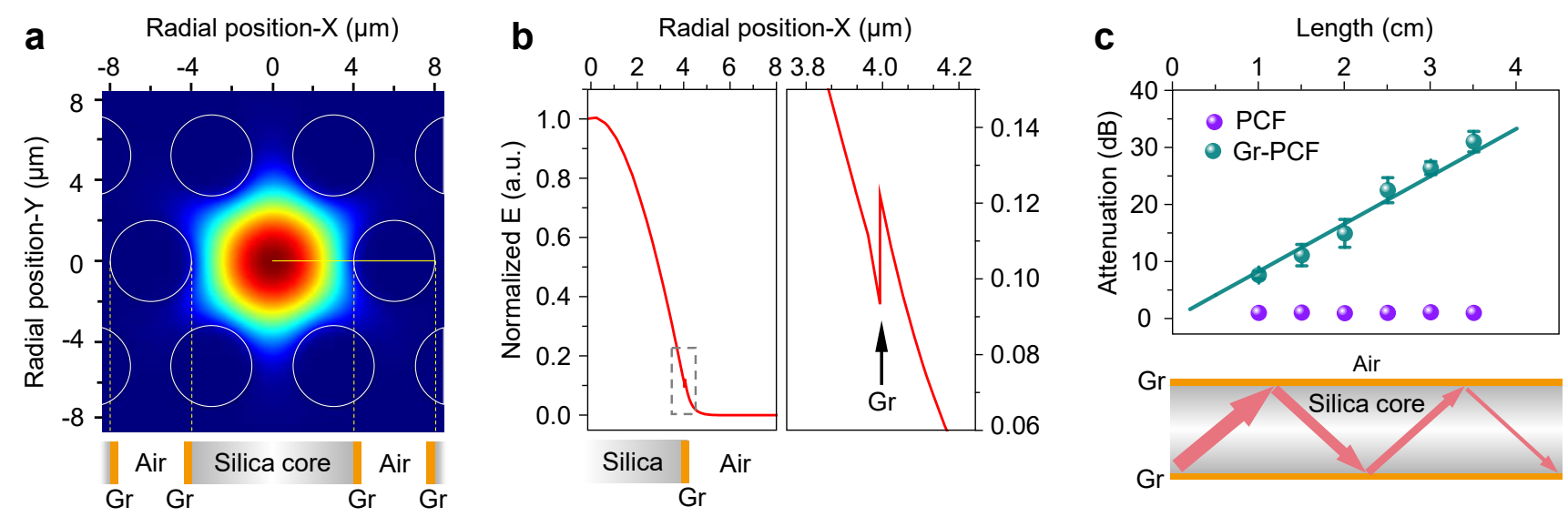

Fig.3 
a

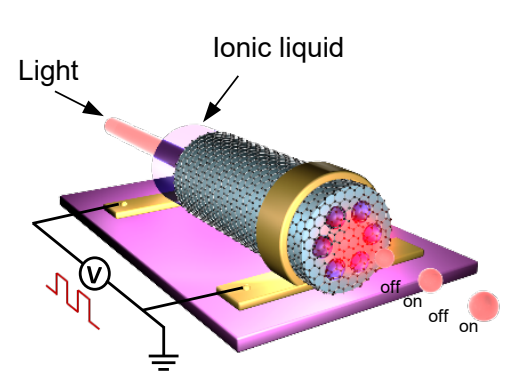

b

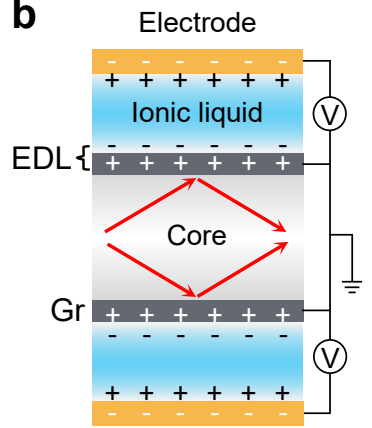

C $\operatorname{Off}\left(E_{\mathrm{F}}<\hbar \omega / 2\right) \quad$ On $\left(E_{\mathrm{F}}>\hbar \omega / 2\right)$

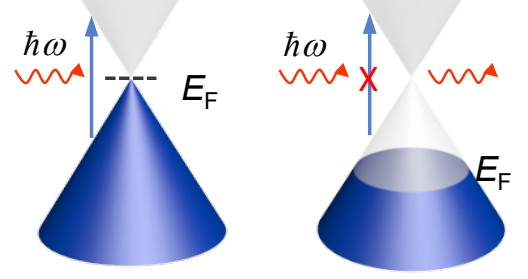

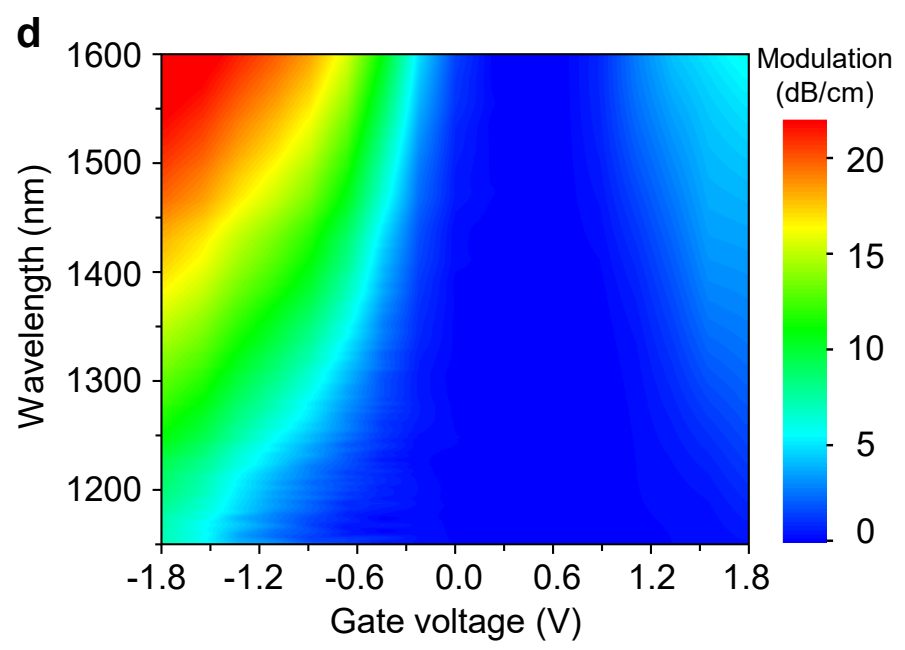

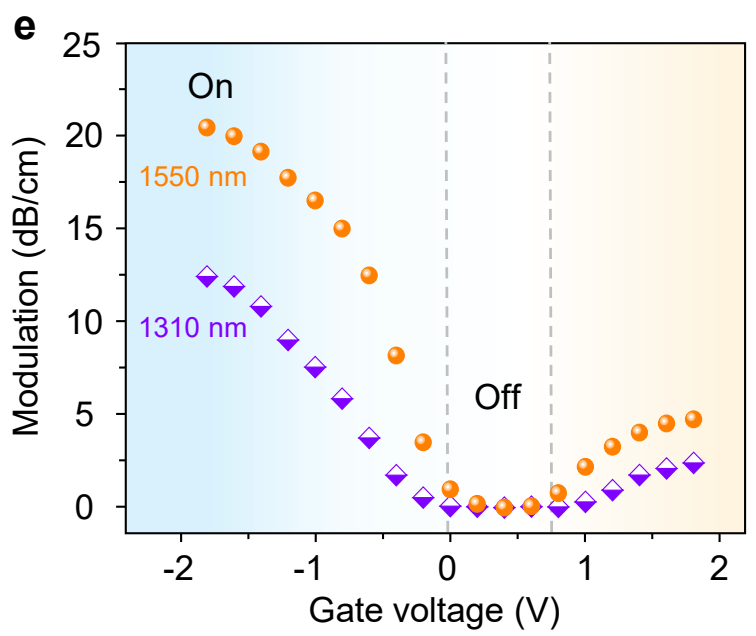

Fig.4 